\title{
COMBINED DES/SD SIMULATON MODEL OF BREAST CANCER SCREENING FOR OLDER WOMEN: AN OVERVIEW
}

\author{
Jeremy J. Tejada \\ SIMCON Solutions, LLC \\ 10817 Trail Weary Drive \\ Austin, TX 78754, USA \\ Kathleen Diehl \\ Department of Surgery \\ Division of Surgery Oncology \\ University of Michigan Health Systems \\ Ann Arbor, MI 48109, USA
}

\author{
Julie S. Ivy \\ James R. Wilson \\ Russell E. King \\ Matthew J. Ballan \\ Michael G. Kay \\ Edward P. Fitts Department of Industrial and \\ Systems Engineering \\ North Carolina State University \\ Raleigh, NC 27695, USA \\ Bonnie C. Yankaskas \\ Departments of Radiology and Epidemiology \\ University of North Carolina \\ 107 Deerfield Trail \\ Chapel Hill, NC 27516, USA
}

\begin{abstract}
We develop a simulation modeling framework for evaluating the effectiveness of breast cancer screening policies for US women of age 65+. We introduce a two-phase simulation approach to modeling the main components in the breast cancer screening process. The first phase is a natural-history model of the incidence and progression of untreated breast cancer in randomly sampled individuals from the designated population. Combining discrete event simulation (DES) and system dynamics (SD) submodels, the second phase is a screening-and-treatment model that uses information about the genesis of breast cancer in the sampled individuals as generated by the natural-history model to estimate the benefits of different policies for screening the designated population and treating the affected women. Based on extensive simulation-based comparisons of alternative screening policies, we concluded that annual screening from age 65 to age 80 is the best policy for minimizing breast cancer deaths or for maximizing quality-adjusted life-years saved.
\end{abstract}

\section{INTRODUCTION}

Breast cancer is one of the most common cancers among North American women, with 232,340 new cases of breast cancer and 39,620 deaths expected in 2013 (American Cancer Society 2013a). The benefits of mammography for middle-aged women are commonly accepted, and much work has been done in evaluating the costs and benefits of screening women in this age group (Mandelblatt et al. 2009; Nelson et al. 2009). On the other hand, there are no well-established screening guidelines for women at least 65 years old (Mandelblatt et al. 2009; Nelson et al. 2009; USPSTF 2009). Furthermore, clinical trials for breast cancer screening have generally not included women who are at least 65 years old; and clinicians do not 
anticipate any clinical trials specific to breast cancer screening in the future (USPSTF 2009). This research addresses the aforementioned gap in breast cancer screening guidelines using simulation.

Section 2 of Tejada et al. (2013a) contains a comprehensive review of previous analytical and simulation-based approaches to the evaluation of breast cancer screening-and-treatment policies. The two simulations developed in our research go beyond the previous work in the following key respects: (i) They use a factor-based method to determine the annual risk of breast cancer for each woman individually; (ii) They use an individualized tumor growth equation in which the tumor growth rate is a function of a woman's age, and the parameters of the equation vary randomly across different women in the system; (iii) They provide a direct linkage between the tumor growth equation and an individualized stochastic process representing the progression of breast cancer through its various stages for each affected woman in the system; (iv) They are calibrated to data from the period 2001-2010, and they project the impacts of both screening and operational policy decisions for the future years 2012-2020; (v) They allow screening policies to be individualized to other important risk factors, not just age; and (vi) They allow the impacts of changes in either screening or operational policies to be evaluated in the same modeling environment.

Figure 1 depicts the overall structure of our two-phase simulation framework. Phase I is the focus of Tejada et al. (2013a), encompassing a natural-history model of the incidence and progression of untreated breast cancer for randomly sampled individuals from the designated population of older US women. The natural-history simulation is a discrete event simulation (DES) model that contains a population growth submodel as well as incidence, progression, and survival submodels. The primary output of the naturalhistory simulation is a database of older women whose untreated breast cancer histories are known; and these histories are critical inputs to the Phase II screening-and-treatment simulation, which is the focus of Tejada et al. (2013b).

The screening-and-treatment simulation integrates DES and system dynamics (SD) modeling techniques so as to represent the following simultaneously: (i) the screening and treatment activities and the resulting progression of health states and incurred costs for each individual in the simulated population; and (ii) the population-level state variables (stocks) and their associated rates of change (flows) that govern the overall operation of the US system for detecting and treating breast cancer.

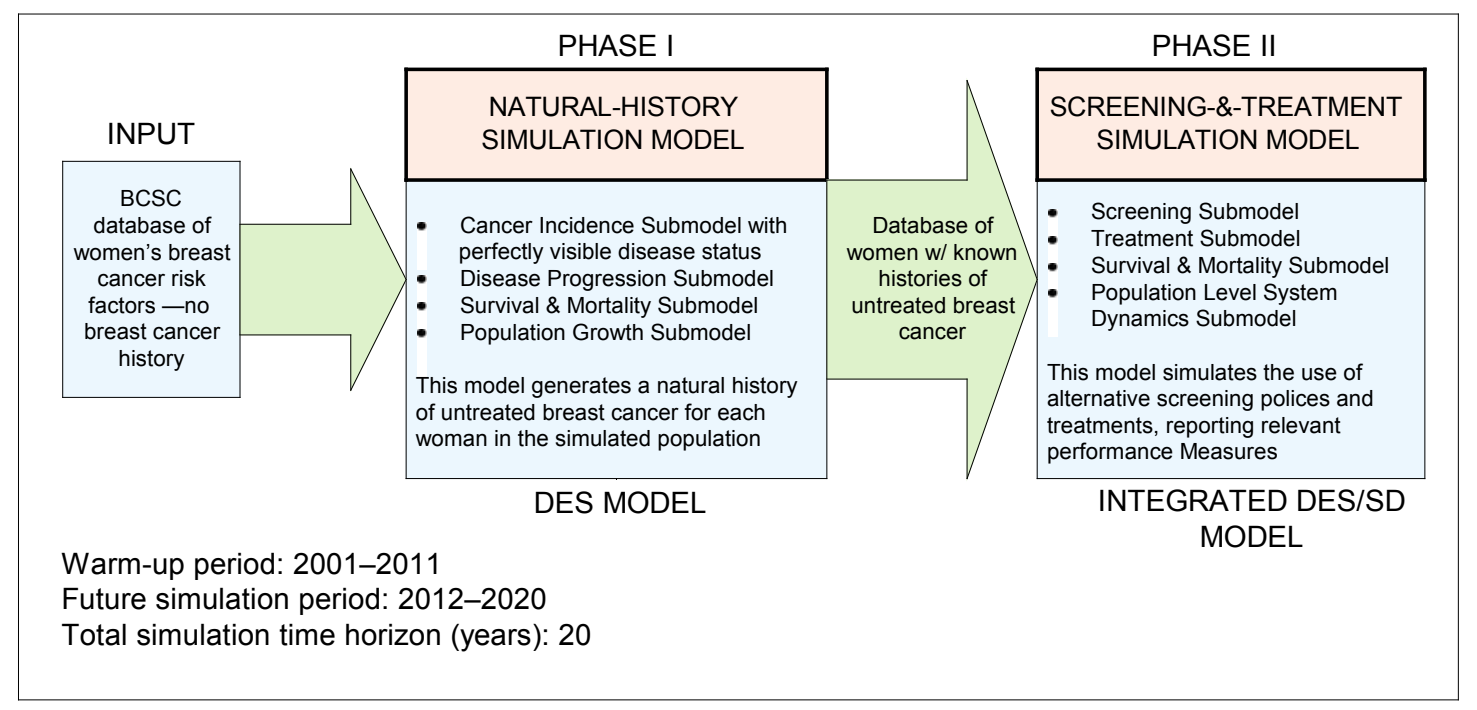

Figure 1: Two-phase simulation approach to evaluating breast cancer screening policies. 
The screening-and-treatment simulation model is composed of interacting submodels that respectively represent screening, treatment, survival and mortality, costing, and population growth. For each woman in the simulated population, the DES submodel represents her associated screening events, diagnostic procedures, and treatment results; however the details of her behavior are subject to the influence of the population-level SD submodel, which encompasses pervasive factors (state variables) that affect her adherence to screening. For both the natural-history and screening-and-treatment simulations, each run spans the period 2001-2020. For each screening policy to be evaluated, the screening-and-treatment simulation calculates key performance measures from the record of detailed activities for each woman in the simulated population.

The remainder of this article is organized as follows. Section 2 covers the natural-history simulation model and its principal submodels. Section 3 contains an overview of the screening-and-treatment simulation model, with special emphasis on the interactions among its principal DES and SD submodels. In Section 4 we summarize the results of an extensive simulation-based comparison of a wide range of screening policies with respect to selected performance measures. In Section 5 we summarize our main findings and recommendations for future work.

\section{NATURAL-HISTORY SIMULATION MODEL}

\subsection{Model Overview}

The natural-history simulation is made up of a number of interacting submodels - namely, the cancer incidence submodel, the disease progression submodel, the survival and mortality submodel, and the population growth submodel. As elaborated below, the most important input to the natural-history simulation is a data set containing information about breast cancer risk factors for individuals in the designated population of US women of age 65+. The Breast Cancer Surveillance Consortium (BCSC 2006, 2009, 2010) provided to us a "de-identified" data set containing breast cancer risk factors for slightly over one million women, where "de-identification" ensures that names, dates of birth, and other identifying information have been removed for the protection of the participants. The BCSC (2010) data set consists of information from seven mammography registries in different locations across the United States, and it is representative of the designated population of US women of age $65+$. For each individual woman in the natural-history simulation, her risk factors are randomly sampled with replacement from this data set when she enters the simulation.

The natural-history simulation establishes a baseline for capturing the benefits of screening and treatment by determining the earliest time that a cancer could be detected for each woman in the simulated population. We assume "perfect visibility" of the disease progression in all women composing the simulated population. We define the ideal of perfect visibility to mean that for each woman, every year her disease status is perfectly observed and verified so that the resulting diagnosis is error free. For the natural-history simulation, we also assume that all cancers remain untreated.

The time-step for both the natural-history and screening-and-treatment simulations is one year-i.e., we simulate all the events for a given year, then we move to the next year and simulate all the events for that year; and this process is repeated until we reach the end of the time horizon in 2020. This approach to the operation of the natural-history simulation makes the most effective use of the available literature, because the logistic regression equations of Barlow et al. (2006) predict a woman's probability each year of being diagnosed with breast cancer as a function of her risk factors after a screening mammogram (breast cancer diagnoses within one year are included); and we use life-tables with breast cancer deaths removed (Rosenberg 2006) to predict the probability of surviving one more year as a function of a woman's current age and birth year. To establish a natural history of untreated breast cancer for each woman in the simulated population, on successive years we compute her one-year probability of being diagnosed with breast cancer and determine if breast cancer would be detected during that year. For each woman in the simulated population, this process continues until one of the following occurs: (i) the woman is still alive, 
either with or without breast cancer, when the simulation stops at the end of the year 2020; (ii) the woman dies from breast cancer before the end of the simulation; or (iii) the woman dies from other causes before the end of the simulation. If based on the Barlow risk equations we determine that the woman develops invasive breast cancer during the simulation, then the cancer is not treated; instead the cancer is allowed to progress until one of the outcomes (i), (ii), or (iii) occurs

Figure 2 shows the possible transitions between health states for each individual woman in the natural-history simulation. Women who are diagnosed with ductal carcinoma in situ (DCIS) are not cancer free; but in the natural-history simulation, we assume that those women do not die from breast cancer based on the evidence provided in the SEER data (SEER 2012a, 2012b).

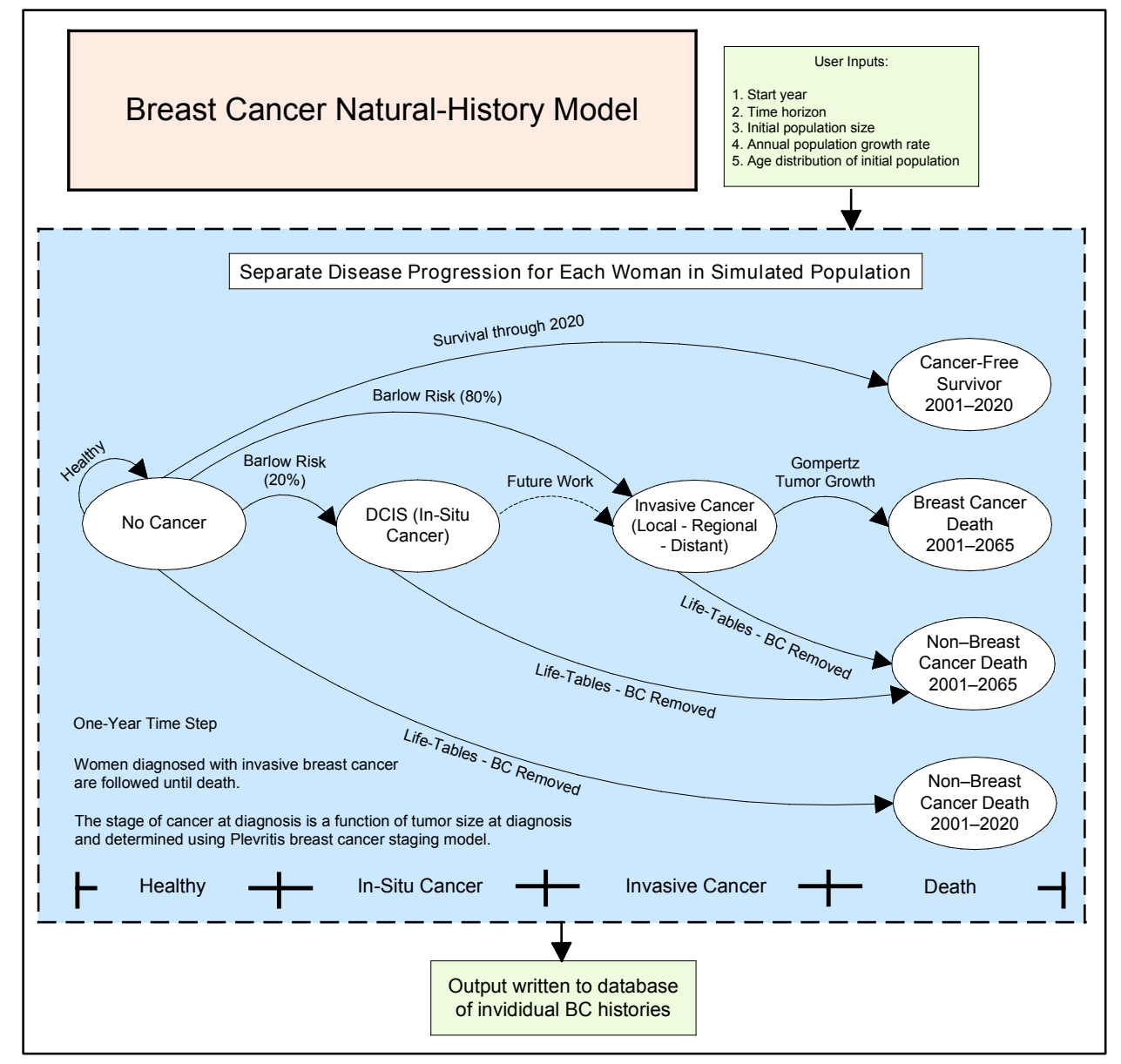

Figure 2: The health-state transitions for the natural-history model.

The risk equations of Barlow et al. (2006) were developed using the BCSC Risk Estimation Data Set, which was collected over the course of a six-year study (BCSC 2006). None of the women in this study had a previous diagnosis of breast cancer, but all of them had undergone breast mammography at some point in the previous five years. Data were collected during the BCSC (2006) study on the following breast cancer risk factors: menopausal status, age, breast density, race, ethnicity, body mass index, family history of breast cancer, previous breast procedures, last mammogram results, surgical menopause, and hormone therapy use.

All the women in our study are at least 65 years old and are assumed to be postmenopausal. In the natural-history simulation, we assume perfect visibility of the disease progression; and in this ideal situa- 
tion, we can use the Barlow risk equations to estimate the probability that a woman with given risk-factor levels will be diagnosed with breast cancer within one year of her mammogram. Because the primary objective of the natural-history simulation is to generate an accurate history of untreated breast cancer for each woman in the simulated population, we simulate an error-free screening mechanism. Barlow et al. (2006) and the documentation for the BCSC Risk Estimation Data Set (BCSC 2006) define "being diagnosed with cancer" in this context as a confirmed diagnosis of breast cancer within one year of the screening mammogram. While the BCSC has data on false positives, there are no false positive results in the BCSC Risk Estimation Data Set. In addition, the effect of false negative results is reduced by waiting one full year after the screening mammogram to make the final determination regarding whether a cancer was actually present. In summary, we believe that the method used to collect the BCSC Risk Estimation Data Set justifies our use of the Barlow risk equations in the natural-history simulation to estimate the probability that a woman will develop breast cancer (invasive or DCIS) within the year following each mammogram.

\subsection{Disease Progression Submodel}

The disease progression submodel consists of a tumor growth equation for tracking tumor size, and a stage progression model for determining the stage of breast cancer as a function of the tumor size at the time of diagnosis. To describe the size of a single breast cancer tumor, we used a Gompertz equation proposed by Norton (1988) that represents the number of cells in the tumor at each point in time. This approach was chosen because there are abundant data suggesting that breast cancer growth in an individual woman can be accurately represented by a general Gompertz equation (Surbone and Norton 1993).

Invasive breast cancer is a progressive disease, and the stage at diagnosis plays a significant role in determining not only the type of treatment used but also the patient's prospects for survival. Breast cancer is typically defined in terms of three stages: local, regional, and distant. The ultimate cause of breast cancer death is the spread of malignant cells to other parts of the body and the resulting destruction of other organs such as the brain and liver. Clearly, both the natural-history simulation and the screening-andtreatment simulation need a method for determining the stage of breast cancer at diagnosis, which is dependent upon the size of the tumor at diagnosis. The larger the tumor, the greater the chance that the cancer has spread to the lymph nodes (regional cancer) or other major organs (distant cancer).

Plevritis et al. (2007) use SEER data (National Cancer Institute 2007) to construct a stochastic process representing the stage progression of breast cancer that allows us to estimate the probability of breast cancer being in the local, regional, and distant stages as a function of tumor diameter. The Plevritis stochastic process fits clinical data reasonably well, and it is easy to incorporate into the natural-history simulation since we have a method for determining the diameter of a woman's breast cancer tumor at diagnosis based on her individualized Gompertz equation for the number of cells in that tumor at that time. In the screening-and-treatment simulation, the stage at diagnosis is used to determine the length of time survived after treatment.

\subsection{Survival and Mortality Submodel}

The "de-identified" BCSC (2010) data set that is randomly sampled in the natural-history simulation does not include information about death ages for women without cancer. To assign a death age for women who do not die of cancer before 2020, we use life-tables provided by Rosenberg (2006) in which breast cancer has been removed as a cause of death. This table contains data for every birth year in the period 1900-2000. In the natural-history simulation, each year a woman has a probability of death occurring from causes other than breast cancer, and this probability is assigned according to the aforementioned life-tables. For each woman who does not die from breast cancer, we compute her age at death from other causes; and we store this quantity for use in statistics calculations and for use when the same population is re-simulated in the screening-and-treatment simulation. This approach allows the natural-history simula- 
tion to differentiate between breast cancer-related deaths and deaths from other causes, making it possible to calculate life-years saved by using different screening policies, and the number of cancer deaths averted in any given year.

\subsection{Population Growth Submodel}

It is important to account for changes in the size of the simulated population over time. To determine the current rate of growth of the designated population of US women of age 65+, we obtained US Census data and determined the annual percentages of growth in that population for the period 2000-2009 (US Census Bureau 2009). We fitted a linear regression equation to this time series. We started in the year 2000 because we wanted to capture the recently increasing trend in the designated population, and the end of the year 2000 is the beginning of the simulation "warm-up" period. We generated annual increases in the size of the simulated population of US women of age 65+ as follows: after the events of one year (e.g., the first year) have occurred in the simulation and the current year has been advanced (e.g., to the second year), we know the number of women who died in the previous year, the number of women still alive, and the expected size of the simulated population at the end of the current year based on the fitted regression equation. Therefore we can estimate the expected increase in the size of the simulated population for the current year; and the number of women actually entering the simulated population at the beginning of the current year is sampled from a Poisson distribution whose mean is our estimate of the expected population increase.

\section{SCREENING-AND-TREATMENT SIMULATION MODEL}

\subsection{DES Submodels for Screening, Diagnostic Procedures, Treatment, Survival, and Costing}

When the screening-and-treatment simulation model is invoked, a user interface is displayed that enables the user to select values for the primary design variables and run-control parameters using option buttons, check boxes, and drop-down combo boxes as detailed in the Online Supplement to Tejada et al. (2013b). Following the user's specification of the screening policy to be evaluated, women enter the screeningand-treatment simulation exactly as they entered the natural-history simulation. As discussed in Tejada et al. (2013a), individual attributes and cancer histories associated with women who entered the naturalhistory simulation are stored in a database in the order that those individuals entered the natural-history simulation. In the screening-and-treatment simulation, those individuals are then retrieved from the database in the same order and are reassigned their corresponding attributes and cancer histories so that they enter the screening-and-treatment simulation at the same points in simulated time that they entered the natural-history simulation.

We perform 10 runs of the screening-and-treatment simulation for each screening policy to be evaluated; and we use the method of common random numbers (Kelton, Sadowski, and Swets 2010) to sharpen the comparisons between different screening policies. Thus, the same 10 randomly sampled populations used in the natural-history simulation are re-created in the screening-and-treatment simulation; and to each individual in each simulated population, we apply separately each screening policy selected for comparison. This approach enables us to compute more precise point and confidence interval (CI) estimators for the mean differences in performance between selected screening policies.

After her attributes and breast cancer history are initialized at the time she joins the simulated population in the screening-and-treatment model, each woman enters the screening submodel that represents all activities related to detection of breast cancer. The screening submodel implements the selected screening policy, samples the probability of adherence to each screening appointment for each individual, and determines the type of screening, diagnostic, and work-up exams to perform on that individual as required. Whereas false positive results and false negative results can occur for screening exams, diagnostic exams can have false positives but not false negatives; and work-up exams such as biopsies are assumed to be perfect so that they yield only true positives and true negatives. If breast cancer is present in an indi- 
vidual, then as in the natural-history simulation (Tejada et al. 2013a, Section 5.2), the stage of breast cancer at diagnosis is determined according to the stochastic process formulated by Plevritis et al. (2007) to represent the progression of the disease.

The treatment submodel is relatively simple, as the details of treatment are not currently the focus of this research. Only women with a detected breast cancer enter the treatment submodel. Through consultation with breast cancer experts, we estimated the probabilities that such women are treated given their age and the presence of other comorbid diseases. If a woman diagnosed with breast cancer does not receive treatment, then in the screening-and-treatment simulation, her age at death and cause of death are identical to the corresponding outcomes in the natural-history simulation.

The survival submodel only processes women who are correctly diagnosed with breast cancer and are selected for treatment in the treatment submodel. For each woman in the survival submodel, we estimate an age at death resulting from breast cancer based on SEER data (National Cancer Institute 2009) and an age at death resulting from other causes based on breast cancer-adjusted life-tables (Rosenberg 2006); and we use the minimum of these two estimates to assign the woman's age at death and cause of death. In addition to computing the number of life-years saved, the survival submodel computes the number of quality-adjusted life-years (QALYs) saved based on utilities from the breast cancer literature.

Within the screening-and-treatment simulation model, the costing submodel keeps track of the costs incurred for screening exams, diagnostic exams, work-up exams, and treatment of breast cancer. The sum of these costs is used to compute the cost-effectiveness of each alternative screening policy.

\subsection{SD Submodel: Population-Level State Variables}

\subsubsection{Structure and Operation of the Combined DES/SD Simulation}

The purpose of the SD submodel is to represent population-level elements of the screening process, specifically those factors influencing adherence to a given screening policy. It may have been possible to capture these effects using a pure DES approach, but the increased computational complexity of such an approach would have caused excessive run times and thus would have prevented us from effectively using simulation optimization techniques to identify promising screening policies. Adherence to a screening policy is based on a number of factors, some at the population level, such as the amount of congestion at screening facilities, and others at the individual level, such as the presence of other comorbid diseases in each woman.

Figure 3 shows the combined DES/SD causal loop diagram for the screening-and-treatment simulation. The top half of the figure displays characteristics of individual women, which are represented as attributes of the associated entities in the DES submodel; and the bottom half of the figure displays characteristics of the population, which are represented by state variables in the SD submodel. The SD and DES submodels are related through the following: (i) a logistic regression equation for predicting nonadherence to breast cancer screening as a function of key attributes of each individual woman; (ii) the "primary state variables" that directly affect the key attributes in (i); and (iii) "hybrid state variables" that directly affect the operation of the DES submodel. In the causal loop diagram, if component A affects component $\mathrm{B}$, then there is an arrow originating at $\mathrm{A}$ and terminating at $\mathrm{B}(\mathrm{A} \rightarrow \mathrm{B})$. There is also a direction of influence, positive or negative, associated with each arrow. A positive influence means that if the state variable associated with component $\mathrm{A}$ increases, then the state variable associated with component $\mathrm{B}$ also in-

creases $\left(\mathrm{A} \rightarrow_{+} \mathrm{B}\right)$. Negative influence means that if the state variable associated with component $\mathrm{A}$ increases, then the state variable associated with component $\mathrm{B}$ decreases $\left(\mathrm{A} \rightarrow_{-} \mathrm{B}\right)$. 
Tejada, Ivy, Wilson, King, Ballan, Kay, Diehl, and Yankaskas

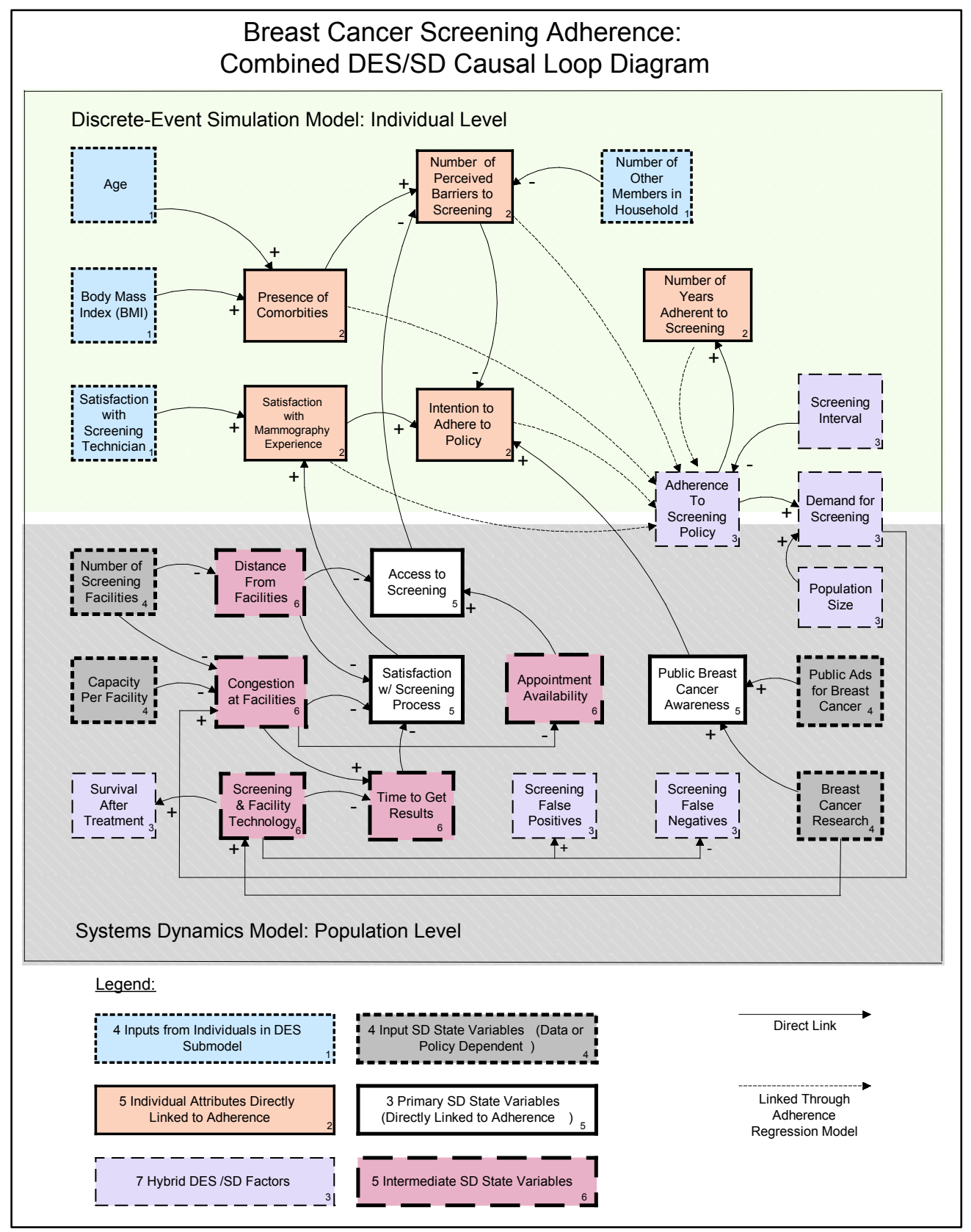

Figure 3: Causal loop diagram for the combined DES/SD model.

\subsubsection{Linkage of DES, SD Submodels via Gierisch Logistic Regression Equation for Nonadherence}

To model adherence to scheduled screening appointments for each woman in the simulated population, Gierisch et al. (2010) formulate a logistic regression equation for predicting the probability that a woman will not attend a scheduled screening appointment as a function of the relevant population-level characteristics as well as the relevant patient-specific attributes. We formulated an SD submodel representing elements of the screening process at the population level. First, we identified the primary state variables directly affecting the attributes of each individual woman that are predictor variables in the logistic regression equation for nonadherence; and then we identified other intermediate state variables that could 
potentially affect those attributes. Some state variables are user inputs, and intermediate state variables are functions of some of the user-assigned state variables or other state variables. Hybrid state variables are defined at the population level but are directly linked to individuals. Moreover, yearly additive changes in the probability of a false negative mammogram depend on the state variable that represents the current level of screening technology.

\section{RESULTS AND DISCUSSION}

In this section we summarize the results of comprehensive experiments with the screening-and-treatment simulation so as to compare the performance of alternative breast cancer screening policies over the period 2012-2020. To make a convincing case that the simulation-generated results for the period 20122020 are a valid representation of what can be expected to happen in the near future under each alternative screening policy, we validated the output of the screening-and-treatment simulation for the period 2001-2011 against SEER breast cancer data for the latter time period. Because of space limitations, the results of this validation are presented in the Online Supplement to Tejada et al. (2013b) and in Tejada et al. (2013c).

With the help of our breast cancer experts, we chose five performance measures as the most important. The five most important performance measures are the following: $M_{1}$, the number of breast cancer deaths during the period 2012-2020; $M_{2}$, the number of QALYs saved by screening during the period 2012-2020; $M_{3}$, the percentage of cancers diagnosed in the distant stage during the period 20122020; $M_{4}$, the cost/QALY saved by screening during the period 2012-2020; and $M_{5}$, the total cost of false positive exams and benign biopsies during the period 2012-2020.

Preventing deaths from breast cancer is the primary objective of screening, and we argue that the number of lives saved (or its complement $M_{1}$ ) is the most important performance measure for a screening policy. Similarly, $M_{2}$ (the number of QALYs saved) is important because it measures not only the years of life saved but also the quality of those additional years of life accumulated over the entire population.

In scenarios involving different overall objectives, we sought to optimize the corresponding performance measure as the basis for identifying the "best" breast cancer screening policy from a set of alternative policies. Therefore, we solved the following three stochastic optimization problems-

$S_{1}$ : Minimize $M_{4}$ (cost/QALY saved) subject to the following constraints: (i) all screening intervals (including low- and high-risk patients) are restricted to 1, 2, 3, 4, or 5 years; and (ii) the stopping age is restricted to $70,75,80,85,90,95$ or 100 years.

$S_{2}$ : Minimize $M_{1}$ (breast cancer deaths) subject to the following constraints: (i) $M_{4} \leq \$ 50,000$; (ii) all screening intervals (including low- and high-risk patients) are restricted to 1, 2, 3, 4, or 5 years; and (iii) the stopping age is restricted to $70,75,80,85,90,95$ or 100 years.

$S_{3}$ : Maximize $M_{2}$ (QALYs saved) subject to the following constraints: (i) $M_{4} \leq \$ 50,000$; (ii) all screening intervals (including low- and high-risk patients) are restricted to 1, 2, 3, 4, or 5 years; and (iii) the stopping age is restricted to $70,75,80,85,90,95$ or 100 years.

OptQuest for Arena (Kelton, Sadowski, and Swets 2010) was used to identify the five best screening policies for each problem; then paired Student's $t$-tests were performed to identify the policy or group of policies that can be declared statistically best in terms of the associated performance measure.

When the objective for the period 2012-2020 is to minimize breast cancer deaths (problem $S_{2}$ ) or to maximize QALYs saved (problem $S_{3}$ ), among the thousands of policies tested we found that the following five screening policies are best (where all policies have a starting age of 65 years): $P_{1}$, annual screening stops at age 80; $P_{2}$, annual screening stops at age 75; $P_{3}$, annual screening for the top $10 \%$ in terms of risk, biennial screening for everyone else, and screening stops at age $80 ; P_{4}$, annual screening for the 
top 5\% in terms of risk, biennial screening for everyone else, and screening stops at age 80; and $P_{5}$, biennial screening stops at age 80 . In addition to policies $P_{1}$ through $P_{5}$, our performance evaluation includes the screening guidelines of the American Cancer Society (ACS) and the US Preventive Services Task Force (USPTF) as follows: (a) policy ACS specifies annual screening for all women of age 65+; and (b) policy USP specifies biennial screening for women from age 65 to age 74 . For problem $S_{2}$, Table 1 summarizes the results (i.e., the sample averages across 10 replications) for the main performance measures accumulated over the simulated population, whose size is $0.1 \%$ of the size of the population of US women of age $65+$. Thus the tabulated results must be multiplied by 1000 to yield comparable estimates for the latter population.

Table 1: Main performance measures for top 5 screening policies to minimize breast cancer deaths.

\begin{tabular}{|l|lllll|}
\hline Policy & $\begin{array}{l}\text { Cancer } \\
\text { Deaths } \\
\text { 2012-2065 }\end{array}$ & $\begin{array}{l}\text { QALYs Saved } \\
\mathbf{2 0 1 2 - 2 0 2 0}\end{array}$ & $\begin{array}{l}\text { \% Distant } \\
\text { Stage 2012- } \\
\mathbf{2 0 2 0}\end{array}$ & $\begin{array}{l}\text { Cost/QALY } \\
\text { Saved } \\
\mathbf{2 0 1 2 - 2 0 2 0}\end{array}$ & $\begin{array}{l}\text { Cost of False } \\
\text { Positives } \\
\mathbf{2 0 1 2 - 2 0 2 0}\end{array}$ \\
\hline $\mathbf{P}_{\mathbf{1}}$ & 538.4 & 1139.9 & 23.5 & $\$ 47,990$ & $\$ 10,042,405$ \\
$\mathbf{P}_{\mathbf{2}}$ & 552.8 & 1058.2 & 28.2 & $\$ 39,182$ & $\$ 8,271,523$ \\
$\mathbf{P}_{\mathbf{3}}$ & 563.1 & 729.4 & 30.1 & $\$ 47,320$ & $\$ 5,009,230$ \\
$\mathbf{P}_{\mathbf{4}}$ & 564.4 & 721.3 & 29.9 & $\$ 48,062$ & $\$ 4,995,410$ \\
$\mathbf{P}_{\mathbf{5}}$ & 560.8 & 744.3 & 30.5 & $\$ 45,030$ & $\$ 4,826,113$ \\
\hline ACS & 536.0 & 1227.2 & 18.3 & $\$ 60,402$ & $\$ 12,424,720$ \\
USP & 560.3 & 686.5 & 33.5 & $\$ 37,952$ & $\$ 4,130,990$ \\
\hline
\end{tabular}

Table 1 summarizes the results for each of the top five screening policies, including the estimated mean values for each of the five primary performance measures and the associated ranking of each policy with respect to each performance measure. Note that the sum of ranks in provides a rough overall measure of the performance of each screening policy when the five main performance measures are taken into account simultaneously. Based on our review of Table 1, we concluded that in terms of breast cancer deaths, QALYs saved, and percentage of cancers diagnosed in the distant stage, the best alternative is policy $P_{1}$. Although policy $P_{1}$ ranks third in cost-effectiveness and last in cost of false positives, these measures are less important; and from a practical standpoint we do not feel the negative impact on lives saved that would result by switching from policy $P_{1}$ to policy $P_{2}$ is justified by the increase in costeffectiveness or by the decrease in cost of false positives that would result from such a switch. For example, from Table 1 we see that on average there are 14.4 fewer cancer deaths with policy $P_{1}$ compared with policy $P_{2}$; however, we must remember that since we are only simulating $0.1 \%$ of the designated population of US women of age $65+$, this translates into an estimate of 14,400 fewer cancer deaths for the designated population over the period 2012-2020. We consider the latter result to be strong evidence that policy $P_{1}$ is preferred in comparison with policy $P_{2}$.

When comparing policies $P_{1}$ through $P_{5}$ with the current guidelines ACS and USP, we concluded that although the USP policy was the most cost effective and had the lowest cost of false positives, it compared unfavorably with policy $P_{1}$ in terms of saving lives. The ACS policy was superior to all the other policies in terms of saving life; however it compared unfavorably with policy $P_{1}$ in terms of cost/QALY saved and cost of false positives. We concluded that policy $P_{1}$ was the most effective compromise between policies ACS and USP. 
Tejada, Ivy, Wilson, King, Ballan, Kay, Diehl, and Yankaskas

\section{CONCLUSIONS AND RECOMMENDATIONS}

From the perspectives of both practical and statistical significance, we concluded that annual screening for all women from age 65 until age 80 was a superior policy in terms of saving lives. Nevertheless, some policy makers may not judge the performance measures $M_{1}$ and $M_{2}$ to be the most important; and one of the primary features of the screening-and-treatment simulation is its ability to evaluate alternative screening policies with respect to almost any relevant performance measure.

Many researchers and practitioners working at the interface between computer simulation and health care systems engineering have strongly supported the idea of effectively integrating the DES and SD methodologies in large-scale health care simulations (Brailsford 2007, 2008); and we believe that our screening-and-treatment simulation can be regarded as a template or guide for how future combined DES/SD simulation models may be designed for other application domains. In addition, the screeningand-treatment simulation provides an approach to modeling a complex disease and the screening and treatment of that disease in a population when several disparate performance measures are of nearly equal importance.

Among the principal directions for future work, special attention should be given to the following: (i) developing a more accurate representation of each woman's attribute for the presence of comorbidities as that attribute depends on her age, health status, and other key medical and socioeconomic variables in the past; (ii) developing a more accurate representation of the way in which each woman's risk factors for breast cancer such as body mass index and family history of breast cancer evolve over time; (iii) developing more accurate representations of the types of treatment for women who are diagnosed with breast cancer and for the survival of those women after treatment as that survival process depends on the type of treatment and the stage of breast cancer at diagnosis; and (iv) formulating a definitive measure of the total effect of false positive exams that can be used to compare alternative screening policies.

\section{ACKNOWLEDGMENTS}

Data collection for this work was supported by the National Cancer Institute-funded Breast Cancer Surveillance Consortium co-operative agreement (U01CA63740, U01CA86076, U01CA86082, U01CA63736, U01CA70013, U01CA69976, U01CA63731, U01CA70040). The collection of cancer data used in this study was supported in part by several state public health departments and cancer registries throughout the United States. For a full description of these sources, please see: http://www.breastscreening.cancer.gov/work/acknowledgement.html.

\section{REFERENCES}

American Cancer Society. 2013a. "Breast Cancer Facts \& Figures 2013." Atlanta, Georgia: American Cancer Society. http://www.cancer.org/research/cancerfactsstatistics/cancerfactsfigures2013/index 2013/index [accessed June 29, 2013].

American Cancer Society. 2013b. "Breast Cancer: Early Detection." Atlanta, Georgia: American Cancer Society. http://www.cancer.org/cancer/breastcancer/moreinformation/breastcancerearlydetection/ breast-cancer-early-detection-acs-recs [accessed October 21, 2012].

Barlow, W. E., E. White, R. Ballard-Barbash, P. M. Vacek, L. Titus-Ernstoff, P. A. Carney, J. A. Tice, D. S. M. Buist, B. M. Geller, R. Rosenberg, B. C. Yankaskas, and K. Kerlikowske. 2006. "Prospective Breast Cancer Risk Prediction Model for Women Undergoing Screening Mammography." Journal of the National Cancer Institute 98 (17): 1204-1214.

Brailsford, S. C. 2007. "Tutorial: Advances and Challenges in Healthcare Simulation Modeling." In Proceedings of the 2007 Winter Simulation Conference, edited by S. G. Henderson, B., Biller, M.-H. Hsieh, J. Shortle, J. D. Tew, and R. R. Barton, 1436-1448. Piscataway, New Jersey: Institute of Electrical and Electronics Engineers. 
Brailsford, S. C. 2008. "System Dynamics: What's in It for Healthcare Simulation Modelers." In Proceedings of the 2008 Winter Simulation Conference, edited by S. J. Mason, R. R. Hill, L. Mönch, O. Rose, T. Jefferson, and J. W. Fowler, 1478-1483. Piscataway, New Jersey: Institute of Electrical and Electronics Engineers.

Breast Cancer Surveillance Consortium (BCSC). 2006. "Risk Model Data Set Documentation." Bethesda, Maryland: National Cancer Institute. http://breastscreening.cancer.gov/rfdataset/rmdataset_docs.pdf [accessed October 21, 2012].

Breast Cancer Surveillance Consortium (BCSC). 2009. "Performance Measures for 1,960,150 Screening Mammography Examinations from 2002 to 2006 by Age-Based on BCSC Data as of 2009." Bethesda, Maryland: National Cancer Institute. http://breastscreening.cancer.gov/data/performance/ screening/2009/perf_age.html [accessed October 21, 2012].

Breast Cancer Surveillance Consortium (BCSC). 2010. "Private Individualized Data Set with Risk Factor Attributes of US Women Undergoing Mammography." Bethesda, Maryland: National Cancer Institute.

Gierisch, J. M., J. A. Earp, N. T. Brewer, and B. K. Rimer. 2010. "Longitudinal Predictors of Nonadherence to Maintenance of Mammography." Cancer Epidemiology, Biomarkers \& Prevention 19:1103-1111.

Kelton, W. D., R. P. Sadowski, and N. B. Swets. 2010. Simulation with Arena. 5th ed. New York: The McGraw-Hill Companies.

Mandelblatt, J. S., K. A. Cronin, S. Bailey, D. A. Berry, H. J. de Koning, G. Draisma, H. Huang, S. J. Lee, M. Munsell, S. K. Plevritis, P. Ravdin, C. B. Schechter, B. Sigal, M. A. Stoto, N. K. Stout, N. T. van Ravesteyn, J. Venier, M. Zelen, and E. J. Feuer. 2009. "Effects of Mammography Screening under Different Screening Schedules: Model Estimates of Potential Benefits and Harms." Annals of Internal Medicine 151:738-747.

National Cancer Institute. 2007. "Surveillance Epidemiology and End Results." Atlanta, Georgia: National Cancer Institute. http://seer.cancer.gov/ [accessed October 21, 2012].

National Cancer Institute. 2009. "Effect of Screening on Breast Cancer Mortality." Atlanta, Georgia: National Cancer Institute.

Nelson, H. D., K. Tyne, A. Naik, C. Bougatsos, B. K. Chan, and L. Humphrey. 2009. "Screening for Breast Cancer: An Update for the U.S. Preventive Services Task Force." Annals of Internal Medicine 151 (10): 727-737.

Norton, L. 1988. “A Gomertzian Model of Human Breast Cancer Growth." Cancer Research 48:70677071.

Plevritis, S. K., P. Salzman, B. M. Sigal, and P. W. Glynn. 2007. "A Natural History Model of Stage Progression Applied to Breast Cancer." Statistics in Medicine 26:581-595.

Rosenberg, M. A. 2006. "Competing Risks to Breast Cancer Mortality." Journal of the National Cancer Institute Monographs 36:15-19.

SEER 2012a. "Relative Survival by Survival Time, by Cancer Site, Ages 65-74, All Races, Female, 1988-2008: Breast (in situ), Female." http://seer.cancer.gov/faststats/ [accessed October 30, 2012].

SEER 2012b. "Relative Survival by Survival Time, by Cancer Site, Ages 75+, All Races, Female, 19882008: Breast (in situ), Female." http://seer.cancer.gov/faststats/ [accessed October 30, 2012].

Surbone, A. and L. Norton. 1993. "Kinetic Concepts in the Treatment of Breast Cancer." Annals of the New York Academy of Science 698:48-62.

Tejada, J. J., J. S. Ivy, R. E. King, J. R. Wilson, K. Diehl, and B. C. Yankaskas. 2013a. "Combined DES/SD Model of Breast Cancer Screening for Older Women, I: Natural-History Simulation. IIE Transactions in review. http://jjtejada.files.wordpress.com/2013/07/uiie-3090r1-pap.pdf [accessed July 12, 2013].

Tejada, J. J., J. S. Ivy, R. E. King, J. R. Wilson, M. J. Ballan, M. Kay, K. Diehl, and B. C. Yankaskas. 2013b. "Combined DES/SD Model of Breast Cancer Screening for Older Women, II: Screening-and- 
Tejada, Ivy, Wilson, King, Ballan, Kay, Diehl, and Yankaskas

Treatment Simulation." IIE Transactions in review. http://jjtejada.files.wordpress.com/2013/07/ pap2r1-7-10.pdf [accessed July 12, 2013].

Tejada, J. J., J. S. Ivy, R. E. King, J. R. Wilson, M. J. Ballan, M. Kay, K. Diehl, and B. C. Yankaskas. 2013c. "Calibration, Validation, and Analysis of a Combined DES/SD Model of Breast Cancer Screening for Older Women." Technical report, Fitts Department of Industrial and Systems Engineering, North Carolina State University, Raleigh, North Carolina. http://jjtejada.files.wordpress.com/ 2013/02/paper-3-version-1-mb-jt-2-5.pdf [accessed July 13, 2013].

US Census Bureau. 2009. "Population Estimates.” http://www.census.gov/popest/ [accessed October 21, 2012].

US Preventive Services Task Force (USPSTF). 2009. "Screening for Breast Cancer: U.S. Preventive Services Task Force Recomendation Statement." Annals of Internal Medicine 151 (10): 716-726.

\section{AUTHOR BIOGRAPHIES}

JEREMY J. TEJADA is the founder and President of SIMCON Solutions, LLC. He is a member of Alpha Pi Mu, Tau Beta Pi, INFORMS, and IIE. His e-mail address is jjtejada@simcon-solutions.com, the company web address is simcon-solutions.com, and his personal web address is jjtejada.wordpress.com.

JULIE S. IVY is an associate professor and Fitts Faculty Fellow in the Edward P. Fitts Department of Industrial and Systems Engineering at North Carolina State University. She is a member of IIE and INFORMS. Her e-mail address is jsivy@ncsu.edu.

RUSSELL E. KING is the Edward P. Fitts Distinguished Professor in the Edward P. Fitts Department of Industrial and Systems Engineering at North Carolina State University, where he is also Director of the Furniture Manufacturing and Management Center. He is a member of INFORMS and he is a Fellow of IIE. His e-mail address is king@ncsu.edu.

JAMES R. WILSON is a professor in the Edward P. Fitts Department of Industrial and Systems Engineering at North Carolina State University. He is a member of ACM, ASA, ASEE, and SCS; and he is a Fellow of IIE and INFORMS. His e-mail address is jwilson@ncsu.edu, and his Web page is www.ise.ncsu.edu/jwilson.

MATTHEW J. BALLAN is a Ph.D. student in the Edward P. Fitts Department of Industrial and Systems Engineering at North Carolina State University. He is a member of Alpha Pi Mu, Tau Beta Pi, and IIE. His e-mail address is mjballan@ncsu.edu.

MICHAEL G. KAY is an associate professor in the Edward P. Fitts Department of Industrial and Systems Engineering at North Carolina State University. He is a member of IIE and INFORMS. His e-mail address iskay@ncsu.edu.

KATHLEEN M. DIEHL is an associate professor of surgery at The University of Michigan. She is a member of the ACS, AMA, SSO, ASCO, ASBD, and ASBS. Her email address is kdiehl@umich.edu, and her web address is http://www.uofmhealth.org/profile/416/kathleen-mary-diehl-md

BONNIE C. YANKASKAS is Professor Emeritus, Department of Radiology and Adjunct Professor in the Department of Epidemiology, University of North Carolina. Her email address is bcy@med.unc.edu. 